\title{
Producción científica educativa, redes de autores y enfoques temáticos: Caso Universidad del Atlántico
}

\section{Educational scientific production, authors" network, and thematic approach: the University of Atlántico}

\author{
José Hernando Ávila-Toscano \\ Universidad del Atlántico, Barranquilla, Colombia \\ Leonardo Vargas-Delgado \\ Universidad del Atlántico, Barranquilla, Colombia \\ Kelly Patricia Oquendo-González \\ Colegio Americano, Barranquilla, Colombia \\ Universidad del Atlántico, Barranquilla, Colombia
}

\section{Resumen}

Introducción: la producción científica en formato de artículo es un mecanismo predilecto para la difusión de resultados investigativos, por medio de ellos se genera impacto académico siendo un indicador relevante en la evaluación de los centros de investigación y universidades. Objetivo: analizar la producción de artículos de investigadores educativos en una universidad colombiana, explorando además las redes de cooperación entre autores y la identificación de los campos temáticos privilegiados en materia educativa. Método: a través de un diseño ex post facto se aplicó análisis de redes sociales en la revisión de artículos científicos. Resultados: se identificó preferencia por revistas de índices regionales, redes de coautoría con pocos intermediarios y una diversidad de áreas de estudio que resaltan el análisis contextualizado de la educación en temas como matemáticas étnicas, literatura, formación y capacitación del profesorado. Discusión y Conclusiones: se discute la necesidad de fomentar la colaboración entre autores y la búsqueda de integración conceptual como estrategias importantes para aumentar la calidad investigativa en educación.

Palabras clave: artículo científico, redes de coautoría, educación, campo de conocimiento.

\begin{abstract}
Introduction: scientific production of articles is the preferred mechanism for disseminating research results. Through these results, academic impact is generated, being a relevant indicator in the assessment of research centers and universities. Objective: to analyze the production of educational research articles in a Colombian university, by exploring cooperation networks among authors and identifying favored thematic fields in terms of education. Method: through an ex post facto design, social networks were assessed and added in the review of scientific articles. Results: Preference over regional index journals was identified in addition toco-authorship networks with few intermediaries and a diversity of study areas that emphasize the contextualized analysis of education in subjects such as ethno mathematics, literature, and teachers' education and training. Discussion and conclusion: The need to foster cooperation among authors and the search for concept integration as important strategies to increase research quality in education is discussed.
\end{abstract}

Keywords: scientific article, co-authorship networks, education, knowledge field.
Open Access:

E-ISSN: $2665-2420$ ISSN: $0124-2121$

ARTÍCULO RESULTADO DE INVESTIGACIÓN Copyright (c) 2020 By Educación y Humanismo

Editor:

Dhayana Fernández Matos Universidad Simón Bolívar

Recibido: 17-02-2020

Aceptado: $28-05-2020$ En línea desde: 02-07-2020

DOI: 10.17081/eduhum. 22. 39.37 


\section{Introducción}

Las investigaciones sobre producción científica cobran mucha importancia en la actualidad debido al fuerte impacto que los estudios bibliométricos y cienciométricos han tenido, puesto que permiten la organización de los sectores científicos por medio de las fuentes bibliográficas, así como la aplicación de estrategias para identificar autores, las relaciones que poseen y la tendencia de sus investigaciones (Spinak, 2001). Estos estudios también resultan relevantes en el análisis del nivel de interdisciplinariedad del conocimiento (Porter \& Rafols, 2009), la descomposición de la literatura científica en disciplinas y subdisciplinas (Leydesdorff \& Rafols, 2009), el análisis de la similitud y conexión entre diferentes áreas de la ciencia (Silva, Viana, Travencolo \& Costa, 2011), la visibilidad de los autores en plataformas digitales (Corchuelo, 2018), entre otros.

El auge de estos estudios también es concomitante con el crecimiento de la producción colombiana, dado que el país ha pasado de tener bajos niveles en la generación de material científico (Aldana, 2010), a registrar aumentos en las contribuciones investigativas a lo largo de los últimos años (Maz-Machado et al., 2016). En esta tarea, las instituciones de educación superior son las que encabezan la producción de documentos científicos (Aldana, 2010; Bucheli et al., 2012), por medio de sus grupos de investigación e investigadores. Precisamente, el análisis de la producción generada por los científicos al interior de sus instituciones y centros de desarrollo de la ciencia, representa una tarea necesaria que además de servir para ponderar la calidad del trabajo de los investigadores (Abramo \& D'Angelo, 2014), facilita generar dinámicas de acceso a recursos para el financiamiento de la ciencia (Brew, Boud, Namgung, Lucas \& Crawford, 2016; Miguel, Chinchilla-Rodríguez, González \& De Moya, 2012), construir redes nacionales e internacionales y crear circuitos de colaboración institucional y entre autores (Shin \& Cummings, 2010).

Aparte de la productividad en cuanto a número de publicaciones y contribución general de los autores a las mismas, dos elementos adicionales son relevantes en este tipo de análisis: la construcción de redes de colaboración y la generación de mapas de conocimiento. En relación con el primer aspecto, la colaboración científica es esencial en toda actividad profesional de un investigador, en tanto el contacto entre autores e instituciones contribuye con la internacionalización y permite acceder a recursos de otros investigadores y grupos, incluso, a aprovechar su prestigio (González-Alcaide \& GómezFerri, 2014; Russel, Ainsworth \& Narváez-Berthelemot, 2006; Puuska, Muhonen \& Leino 2014).

A esto se suma que la colaboración nacional e internacional permite abarcar distintos problemas sociales desde enfoques y perspectivas diversas, además aumenta el espíritu crítico y la comunicación entre la comunidad científica (González-Alcaide et al., 2008; 
Luna-Morales, 2012; Sancho, Morillo, De Filippo, Gómez \& Fernández, 2006). Este trabajo colaborativo favorece el desarrollo de las sociedades gracias a la multivariedad de culturas y conocimientos (Luna-Morales, 2012). Por esta razón los organismos reguladores del desarrollo de la ciencia en el mundo reconocen la importancia que tiene cooperaren el campo investigativo, lo cual inspira al desarrollo de investigaciones con más calidad y relevancia (Sancho et al., 2006).

El segundo elemento corresponde a la identificación de mapas de conocimiento derivados de la producción científica, los cuales son una herramienta que permite visualizar y localizar el conocimiento, y que además está muy relacionada con las redes de colaboración. La utilidad de esta herramienta radica en la capacidad para representar la actividad científica, permitiendo descubrir con suficiencia la estructura de una disciplina según el tipo de información analizada (Guzmán \& Trujillo, 2013), esto deja conocer subcampos, ejes temáticos o dominios dentro de una dimensión evaluada, distinguiendo además entre temas principales y auxiliares (He, 1999).

Ahora bien, la aplicación de este tipo de análisis ha ofrecido contribuciones diversas en el estudio de la producción y estado de las ciencias de la educación, si bien la evidencia se enfoca en líneas formativas puntuales (Bracho-López et al., 2012; Maz-Machado et al., 2011), sin embargo, resultados recientes en el contexto colombiano han ofrecido datos acerca de la productividad de los grupos de investigación en educación (Ávila-Toscano, Romero-Pérez, Saavedra \& Marenco-Escuderos, 2018), la producción de investigadores hombres y mujeres, sus indicadores de redes colaborativas e institucionales (ÁvilaToscano, Marenco-Escuderos \& Romero-Pérez, 2019), e incluso, se ha descrito un mapa general del campo de conocimiento de la educación colombiana. Respecto a este último, los datos recientes señalan que entre el periodo 2006-2015, la investigación educativa publicada en artículos científicos por investigadores adscritos a grupos de investigación colombianos se ha enfocado en el análisis de la aplicación de la tecnología en la educación, así como en el abordaje de técnicas y métodos en el proceso enseñanzaaprendizaje. También se señala un notorio enfoque de desarrollo humano que permea gran parte de la investigación educativa y un carácter interdisciplinario de la misma (ÁvilaToscano, Romero-Pérez, Marenco-Escuderos \& Saavedra-Guajardo, 2018).

Sintetizando, las universidades, sus centros de estudios o facultades y los diferentes grupos de investigación, requieren de una revisión periódica de sus dinámicas investigativas y las temáticas abordadas. Los estudios bibliométricos y cienciométricos resultan óptimos en este empeño por permitir la caracterización de los productos y la actividad académica de la que se desprenden, además contribuyen a reconocer la posición que los autores y las instituciones ocupan dentro de la diáspora científica, otorgando amplios informes que permiten tomar la iniciativa al buscar estrategias que mejoren las formas de interacción académica. De manera puntual, en la investigación de temas educativos es fundamental tener conocimiento acerca de la tendencia de las investigaciones realizadas (Papadimitriou \& Kidman, 2012), los enfoques planteados o las 
conexiones entre las propuestas investigativas y teóricas y la delimitación del campo de estudio, y a partir de estas, crear nuevas líneas investigativas enfocadas en el desarrollo de ciencia, tecnología e innovación aplicadas a la educación.

Precisamente, el objetivo de este artículo abarca todos los temas antes descritos; se enfoca en analizar los indicadores de producción científica de grupos de investigación educativa, puntualizando en el caso de la Universidad del Atlántico, al tratarse de una de las instituciones de educación superior más importantes del Caribe colombiano. El análisis se enfoca tanto en caracterizar la producción investigativa, como en generar mapas de conocimiento y redes de colaboración entre investigadores.

\section{Método}

\section{Unidad de análisis}

A través de un estudio ex post facto, se realizó el análisis de la producción de nueve grupos de investigación adscritos a la Facultad de Educación de la Universidad del Atlántico (Colombia), enfocándose en particular sobre los artículos publicados entre enero de 2009 y mayo de 2018, debidamente registrados en los aplicativos digitales (GrupLAC) de cada grupo de investigación reconocido por Colciencias.

\section{Variables de análisis}

Dos conjuntos de variables fueron considerados en este estudio, por un lado, se analizaron indicadores bibliométricos que permiten un análisis descriptivo de la producción. Dentro de los indicadores se incluyó el tipo de artículo, diferenciando entre los incluidos en bases de Web of Science (WoS) y SCOPUS y los publicados en revistas incluidas en Bases Bibliográficas con Comité Científico (BBCC) (Colciencias, 2017); además se registró si esta producción era endógena (publicada en revistas de la misma universidad) o exógena. Así mismo, tras la revisión y lectura de cada artículo se identificó su enfoque (cualitativo, cuantitativo, mixto), el tipo de investigación (aplicada, experimental o cuasiexperimental, teórica) y el tipo de cooperación entre autores (sin cooperación, cooperación intrainstitucional, interinstitucional, internacional) (GonzálezAlcaide et al., 2008). Adicionalmente, se identificó número de autores, número de firmas y género de autores.

El segundo conjunto de datos corresponde a la identificación de autores sobresalientes, las estructuras de cooperación entre autores y las redes temáticas. Para ello, se aplicó análisis de redes enfocado en medidas de centralidad, es decir, aquellas que determinan la importancia relativa de los autores. Esto se cumplió mediante el cálculo de los indicadores de grado nodal (relaciones entre autores en la red; número de actores que establece colaboración); Cercanía (indica en qué medida un autor se integra con otros), Intermediación (define si un autor actúa de intermediario entre otros), Centralización 
(identifica si las relaciones en la red se concentran en un conjunto específico de autores).

Así mismo, se analizó el nivel de cohesión de la red de autores considerando el nivel de densidad (totalidad de vínculos establecidos entre todos los autores), Reciprocidad (medida en que los vínculos son recíprocos en una red) y las Distancias geodésicas (rutas requeridas para que los actores cooperen), incluyendo el nivel de compactación o fragmentación de la red de autores. Finalmente, se calculó el indicador de clúster (subconjuntos existentes dentro de una misma red), que permitió identificar los diferentes grupos temáticos.

\section{Procedimiento y análisis de datos}

Los artículos incluidos en este estudio fueron revisados en los aplicativos GrupLAC de cada grupo de investigación, y posteriormente se consultaron en los archivos de cada revista, verificando su clasificación y vigencia, de esta forma se pudo diferenciar si el producto correspondía a artículo incluido en WoS/Scopus o en otra BBCC.

La información correspondiente a cada producto fue cargada en una base de datos para su posterior análisis descriptivo. Así mismo, se construyeron dos matrices de redes, una con todos los autores firmantes en los artículos analizados y otra con las palabras clave. La primera fue una matriz cuadrada de código binario $(1,0)$ que define la cooperación o no entre autores y fue creada con el software Ucinet 6.391 (Borgatti, Everett \& Freeman, 2002), con el cual también se cumplió el cálculo de los indicadores de la red.

A esta red se le adicionaron atributos relacionados con los autores, incluyendo sexo, país de procedencia, nivel de formación (pregrado, maestría, doctorado) y categoría como investigador (senior, asociado, junior, otro integrante) de acuerdo con la clasificación de Colciencias. Para recabar esta información se accedió a los currículos públicos de los investigadores.

Estos atributos se correlacionaron con las características relacionales de la red de autores por medio del procedimiento denominado correlación QAP (Quadraticas Sigment Procedure) propuesto por Hubert y Schultz (1976), que facilita la identificación del grado de asociación entre diferentes variables. Dicho procedimiento se basa en calcular el coeficiente de correlación de dos matrices observadas, y posteriormente se compara si los actores se relacionan en las dos matrices. El cálculo arroja varios estadísticos siendo el más conocido el coeficiente de correlación de Pearson. Tras identificar qué atributos mostraron correlaciones significativas con la red de autores, se procedió a calcular un modelo de regresión múltiple (Double Dekker Semipartialiting- MRQAP) aplicable al procedimiento QAP.

Finalmente, la red de palabras clave se generó con una matriz de borde vertical con el software NodeXLTemplate Pro (CodePlex, 2013), a través del cual se cumplió con el 
cálculo de los clústeres y la generación de un grafo para la visualización de la red, incluyendo sus correspondientes datos estructurales (número de términos y conexiones entre ellos, densidad y distancias geodésicas).

\section{Resultados}

\section{Datos bibliométricos}

Se analizaron 55 artículos publicados entre enero de 2009 y mayo de 2018, de esta producción, su gran mayoría corresponde a contribuciones publicadas en revistas incluidas en índices de impacto regional, o en BBCC según la clasificación de Colciencias, (2017) (p. e.: Scielo, RedALyC, LILACS, etc.), con un total de 47 productos $(85,4 \%)$, mientras que solo $8(14,6 \%)$ corresponden a artículos incluidos en las bases de Web of Science (WoS) y SCOPUS. Por su parte, del total de artículos producidos, 16,3\% ( $n=9)$ fueron endógenos, es decir, publicados en revistas editadas por la universidad de origen.

Primó el enfoque cualitativo $(76,4 \%, n=42)$ como el de mayor aplicación dentro de la propuesta metodológica de los artículos trabajados, mientras que los documentos basados en el enfoque cuantitativo correspondieron al 23,6\% $(n=13)$. Así mismo, 74,6\% $(n=41)$ de estos estudios se basaron en investigación aplicada, 9,1\% $(n=5)$ fue desarrollado bajo el paradigma experimental o cuasiexperimental y $16,4 \%(n=9)$ correspondió a investigación teórica.

En relación con la cooperación en el desarrollo de los artículos, los resultados observados son modestos en tanto que, 49\% $(n=27)$ de los artículos fueron desarrollados por único autor. Entre los artículos con cooperación, se identificó una media de 1,5 (de= $1,13)$ instituciones filiadas en los documentos; $20 \%(n=11)$ de los artículos cuenta con cooperación intrainstitucional, $23,7 \%(n=13)$ interinstitucional y solo $7,3 \%(n=4)$ se desarrolló con lazos internacionales.

Los artículos fueron escritos por un total de 69 autores (39 hombres y 30 mujeres) que registran 114 firmas, las mismas se dividen en 71 firmas de hombres (62,3\%) y 43 de mujeres $(37,7 \%)$.La media de autores por contribución fue de 2,07 (de=1,39), con un máximo de 8 autores por artículo, mientras que, según el sexo, la media de autores hombres fue de 1,29 (de=0,97, Mín.=0-Máx.=5) y la de mujeres fue de 0,78 $(d e=0,95$, Mín.=0-Máx.=3). 


\section{Análisis de redes de coautorías}

Se aplicó Análisis de Redes Sociales para comprender la estructura relacional de los vínculos de coautoría tejidos en todos los artículos evaluados. Para ello se calcularon indicadores de centralidad y de cohesión de la red de autores, y posteriormente se probó la relación de la estructura de la red con las características de los autores. En la Tabla 1 se presentan los datos descriptivos de los indicadores de cohesión, centralidad y subagrupaciones.

Tabla 1.

Datos descriptivos de los indicadores de cohesión y centralidad en la red de coautorías.

\begin{tabular}{cccccc}
\hline & Indicador & Valor & de & Mín. & Máx. \\
\hline Centralidad & Grado & 3,07 & 2,89 & 0,00 & 16,00 \\
& Cercanía de entrada & 1,68 & 0,17 & 1,44 & 1,88 \\
& Cercanía de salida & 1,67 & 0,16 & 1,44 & 1,88 \\
Cohesión & Intermediación & 0,13 & 0,66 & 0,00 & 4,17 \\
& Densidad & 0,04 & 0,20 & & \\
& Reciprocidad & 0,99 & & & \\
& Distancia geodésica & 1,66 & & & \\
& Compactación & 0,08 & & & \\
& Fragmentación & 0,91 & & & \\
Subgrupos & Clúster & 33 & & & \\
\hline
\end{tabular}

Fuente: elaboración propia.

Pese a tratarse de una red pequeña $(n=69)$, su indicador de densidad es bajo, denotando poca conexión entre los integrantes; este reducido desempeño cohesivo además se reitera con altos niveles de fragmentación y la identificación de un amplio número de subagrupaciones que en la red alcanza un total de 33 subgrupos con un mínimo de tres actores. Tales datos señalan que no se trata de una red jerarquizada; complementariamente, el análisis de centralización de grado muestra poca concentración del flujo de relaciones cooperativas en un conjunto reducido de autores, con un valor de $19,58 \%$, además, las distancias entre actores son relativamente cortas dado que $34 \%$ de los vínculos está a una distancia geodésica, $65 \%$ está a dos distancias y solo $1 \%$ de los contactos requiere de tres distancias.

Por su parte, la Tabla 2 describe a los autores que sobresalen de acuerdo con sus indicadores de centralidad. En este caso, destaca el papel de Armando Aroca y Antonio Medina como los articulistas con mayor indicador de grado, reportando 16 y 15 conexiones respectivamente, la cual duplica y triplica el reporte de este indicador frente a los demás autores destacados. Estos mismos investigadores destacan por poseer los más elevados niveles de intermediación, de hecho, son solo siete los intermediarios de la red, es decir, a partir de ellos es posible acceder a nuevos lazos cooperativos. 
Tabla 2.

Principales autores de acuerdo con sus indicadores de centralidad.

\begin{tabular}{|c|c|c|c|c|c|c|}
\hline Grado & Valor & Intermediación & Valor & Cercanía & Entrada & Salida \\
\hline Armando Aroca & 16 & Armando Aroca & 190,0 & Armando Aroca & 1,88 & 1,88 \\
\hline Antonio Medina & 15 & Antonio Medina & 172,0 & Elidia Castillo & 1,88 & 1,88 \\
\hline Elidia Castillo & 7 & Roberto Figueroa & 18,00 & Molly Tun & 1,88 & 1,88 \\
\hline Molly Tun & 7 & Sonia Valbuena & 14,00 & Sergio Pou & 1,88 & 1,88 \\
\hline William Flores & 7 & Mirna Bernal & 10,00 & William Flores & 1,88 & 1,88 \\
\hline Sergio Pou & 7 & Francisco Racedo & 4,00 & Leonel Morales & 1,88 & 1,88 \\
\hline Leonel Morales & 7 & María Domínguez & 2,00 & Domingo Yojcom & 1,88 & 1,88 \\
\hline María Elena & 7 & & & María Elena & 1,88 & 1,88 \\
\hline Domingo Yojcom & 7 & & & Hilbert Blanco & 1,87 & 1,87 \\
\hline Sonia Valbuena & 6 & & & Diana Gil & 1,87 & 1,87 \\
\hline Roberto Figueroa & 5 & & & Jhonatan Arenas & 1,87 & 1,87 \\
\hline Francisco Racedo & 5 & & & Mayra Jiménez & 1,87 & 1,87 \\
\hline Edna Gómez & 4 & & & Mónica Rodríguez & 1,87 & 1,87 \\
\hline Nelson Alvis & 4 & & & Lady Correa & 1,87 & 1,87 \\
\hline Elvira Flórez & 4 & & & Norbey Medina & 1,87 & 1,87 \\
\hline Jannina Alvis & 4 & & & Jonathan Cervantes & 1,87 & 1,87 \\
\hline María Domínguez & 4 & & & Lina Álvarez & 1,87 & 1,87 \\
\hline Luis Alvis & 4 & & & Antonio Medina & 1,85 & 1,81 \\
\hline Rafael Sarmiento & 3 & & & María Domínguez & 1,84 & 1,81 \\
\hline Adiela Ruiz & 3 & & & Eduardo Ramos & 1,84 & 1,81 \\
\hline
\end{tabular}

Fuente: elaboración propia.

Las características de los autores (sexo, país de origen, nivel de formación y categoría) fueron analizadas en relación con la estructura de la red de coautorías por medio del análisis de correlaciones QAP, posteriormente, las correlaciones significativas se analizaron mediante regresión múltiple aplicada al procedimiento de asignación cuadrática (MRQAP). Los resultados se aprecian en la Tabla 3.

Tabla 3.

Resultados de correlación QAP y del modelo de Regresión múltiple (Double Dekker Semipartialiting $M R Q A P)$ entre la red de coautorías y los atributos de los autores.

\begin{tabular}{cccccc}
\hline & \multicolumn{3}{c}{ QAP } & \multicolumn{2}{c}{ MRQAP $^{\mathbf{a}}$} \\
\hline & Coautoría & Sexo & Categoría & $\beta$ & $p$ \\
\hline Sexo & 0,00 & & & & \\
Categoría & $-0,00$ & $-0,02$ & & & \\
Formación & $0,08^{*}$ & $-0,02$ & $-0,05$ & 0,06 & $0,01^{*}$ \\
País & $0,14^{*}$ & 0,01 & $-0,06^{*}$ & 0,13 & $0,00^{*}$ \\
\hline
\end{tabular}

${ }^{\mathrm{a}} \mathrm{R}^{2}=0,026, \mathrm{R}_{\text {corregido }}^{2}=0,025, * p<0,05$

Las correlaciones halladas corresponden a los atributos Formación y País, sugiriendo vinculación homofílica entre autores de la misma nacionalidad y nivel formativo, sin embargo, los coeficientes de correlación son pequeños lo que denota asociación débil 
entre las variables. Esto se refrenda con el modelo de regresión en el cual el coeficiente tipificado de mayor envergadura corresponde al atributo País $(\beta=0.13)$, pero el valor $R^{2}$ y su corrección son muy bajos por lo que se opta por descartarlos.

\section{Análisis de campo temático a partir de palabras clave}

La red de palabras clave contenidas en los artículos se analizó por medio del cálculo de clúster, lo que permitió identificar 21 agrupaciones a partir de mínimo 3 términos. Con el fin de identificar grupos de mayor elaboración semántica se definieron como conjuntos principales los constituidos por un mínimo de 10 palabras clave. Esto dejó un total de seis conjuntos semánticos principales cuyas redes se aprecian en la Figura 1.

Figura 1.

Grafo de subagrupaciones semánticas emergidas en el análisis de clúster de palabras clave y sus respectivos datos descriptivos

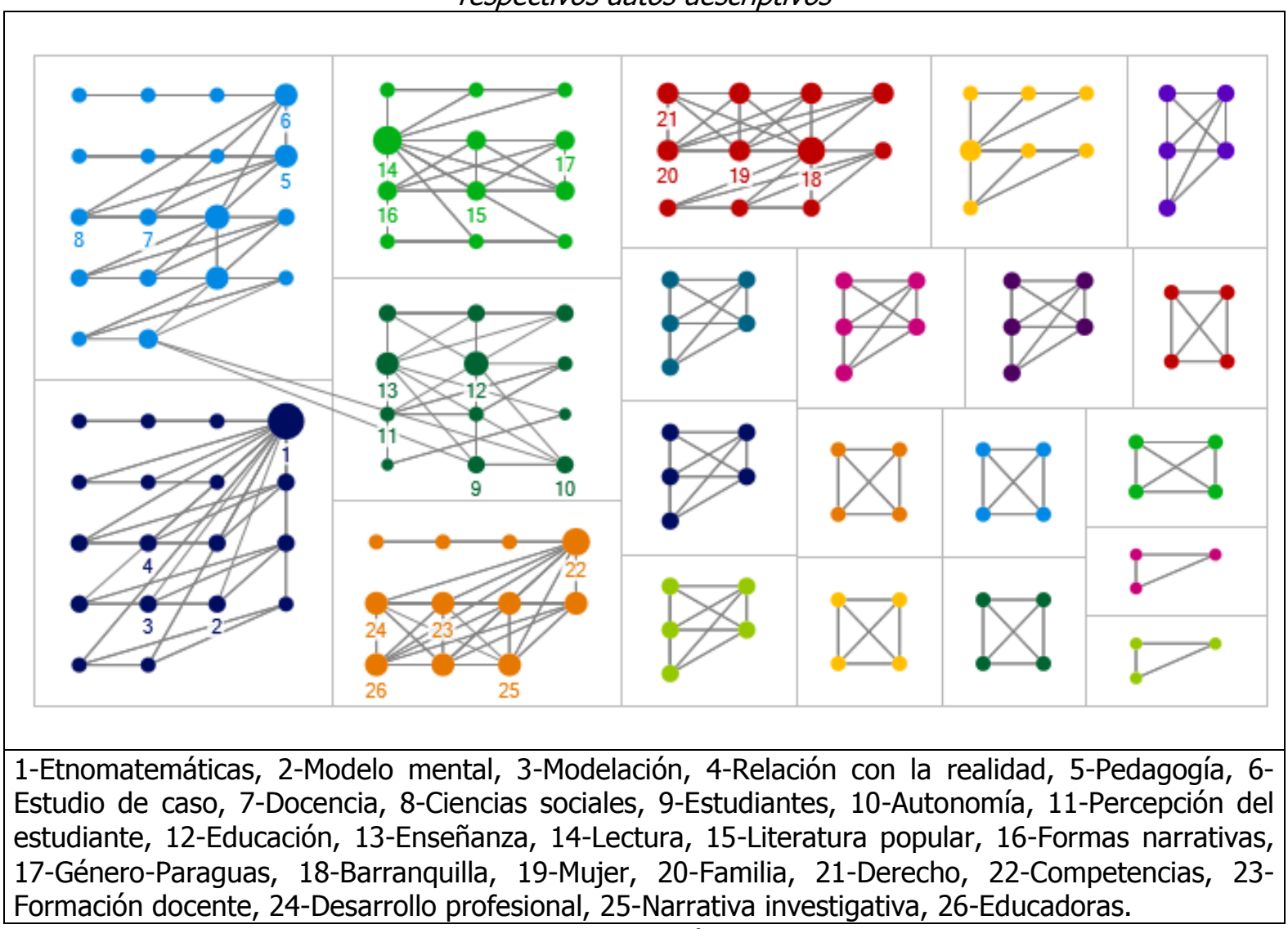

Fuente: elaboración propia.

El grupo 1 se denomina educación matemática en contextos socioculturales, incluye 18 términos con 72 conexiones, con una distancia geodésica promedio de 1,65 y densidad de 0,25 . Este grupo se enfoca preponderantemente en el estudio de las prácticas culturales de la matemática, destacando el abordaje etnomatemático como recurso fundamental en la educación inclusiva en diferentes etnias y culturas, así mismo, incluye saberes geométricos y construcciones matemáticas desde la perspectiva cultural y su estudio 
desde la formación universitaria.

El segundo conjunto temático se denomina Conocimiento pedagógico del docente, también incluye 18 términos con 73 conexiones entre ellos, pero mayor distancia geodésica promedio $(2,15)$ y densidad de 0,25. Este grupo comporta temáticas relacionadas con el conocimiento del profesorado entorno a la educación y a la pedagogía, incluyendo metodologías, políticas, aspectos curriculares e incluso componentes actitudinales de los actores educativos.

El grupo tres se denomina Aplicación de las TIC a la enseñanza, cuenta con 15 palabras y 57 conexiones, promedio de distancia geodésica de 1,71 y densidad de 0,29. Con un nombre muy diciente, este campo aborda la investigación pedagógica y la enseñanza desde la perspectiva del uso y aprovechamiento de las tecnologías de la información y la comunicación.

La denominación Lectura y escritura en el ámbito universitario corresponde a la cuarta agrupación semántica, formada por 12 términos que establecen 54 conexiones, con una densidad mayor a la de los demás grupos $(0,41)$ y 1,46 distancias geodésicas de promedio. Este conjunto temático se concentra en el análisis de las prácticas de lectoescritura de diferentes géneros o narrativas enfocándose en estudiantes de educación superior.

Finalmente, los grupos cinco y seis, ambos con 11 palabras, comparten características similares. El primero se denomina Conflicto social y educación en el Caribe colombiano y se forma a partir de 62 conexiones, con una densidad de 0,56 y un máximo de dos distancias entre términos; este grupo dirige el interés sobre problemáticas propias del conflicto social colombiano y la vulneración de los derechos de niños, niñas y sus familias, haciendo hincapié en el contexto Caribe. Por su arte, el grupo seis, denominado Formación en competencias del docente es el de mayor densidad $(0,62)$ y menor promedio de distancia entre los términos $(1,26)$, hace referencia al estudio de las competencias a considerar en el proceso de formación de nuevos docentes, distinguiendo además un abordaje diferencial enfocado en el género.

Los grupos dos y tres, son los únicos conjuntos semánticos que comparten conexión a partir de los términos "estudiantes" y "autonomía" (ver Figura 1), mientras que en el resto de subagrupaciones las interconexiones se dan de forma interna, es decir, la red semántica se compone de diferentes conjuntos de temas particulares sin relaciones comunes.

\section{Discusión}

Este estudio aborda tres aristas esenciales en el análisis de la producción científica en el campo de la educación, que, para casos puntuales, se enfoca en la producción generada desde la Facultad de Ciencias de la Educación de una universidad. Los tres aspectos de 
análisis son: a) los indicadores bibliométricos de artículos científicos, b) las redes de coautoría y c) el mapa de conocimiento o campo temático. Frente al primer aspecto, los resultados de este estudio muestran un nivel moderado de producción de artículos científicos en tanto la suma de producciones es de 55 documentos, lo que corresponde a un promedio de 5.5 artículos por año y 6.1 artículos por grupo de investigación. Este dato no resulta del todo sorprendente en función de la preferencia histórica de otros formatos para la difusión del conocimiento tanto en el campo educativo como en otras áreas de las ciencias sociales y las humanidades (Hicks, 2004), sin embargo, bien es cierto que en la última década ha venido dándose un aumento en la generación de artículos científicos en todas estas áreas. Puntualmente en Colombia, trabajos recientes han mostrado que la educación es una de las áreas del conocimiento con mayor cantidad de investigadores y publicaciones (Ávila-Toscano et al., 2019), esto conduce a pensar en la importancia de producir nuevo conocimiento cuya difusión se enfoque en el formato de artículo, pues el mismo permite la posibilidad de acceder a múltiples canales de difusión facilitando su apropiación tanto en el plano nacional como internacional.

La mayor parte de estos trabajos se basa en investigación aplicada que obedece a metodología cualitativa, generalmente publicados en revistas científicas incluidas en bases de impacto regional o local, mientras que el número de contribuciones publicadas en revistas incluidas en WoS/SCOPUS es menor. La evidencia sobre el tema coincide en señalar que en disciplinas sociales, humanidades y educación, existe una preferencia por este tipo de revistas (Rosario-Sierra, Sánchez-Perdomo, Herrera-Vallejera \& RodríguezSánchez, 2017) en tanto las mismas funcionan con formatos menos rígidos, permiten la difusión del conocimiento en idioma español, muestran más apertura a metodologías cualitativas (Gantman, 2011) y además son una excelente forma de promover la integración entre autores latinoamericanos lo que constituye una estrategia de fortalecimiento regional (Vélez-Cuartas, Lucio-Arias \& Leydesdorff, 2016).

La discusión sobre el tipo de formato predilecto en disciplinas como la educación y el tipo de formato privilegiado por los sistemas de ciencia y tecnología, está ahora más vigente que nunca, las líneas de análisis en el tema sostienen la necesidad de estimular la publicación en revistas regionales como una forma de atender problemáticas particulares de los contextos latinoamericanos y promover la integración entre autores cuyos temas de interés son comunes (Gantman, 2011, Vélez-Cuartas et al., 2016; Ávila-Toscano et al., 2018), al tiempo, resulta importante (y necesario) generar producción en revistas de alto impacto internacional cuyo formato se premia dentro de los sistemas de ciencia y tecnología como el colombiano, y que además resultan prioritarios para lograr el aumento en los niveles de clasificación de alta calidad de los grupos de investigación (Ávila-Toscano et al., 2018). Pensar en este equilibrio puede resultar provechoso para las ciencias de la educación, en tanto se responde a las lógicas de los sistemas y modelos contemporáneos de producción y circulación del conocimiento, sin desatender al enfoque ideológico intrínseco de esta área encaminado hacia el reconocimiento del valor de la realidad y los fenómenos asociados con el contexto local y regional. 
El segundo enfoque de este trabajo versa sobre las redes de coautoría como mecanismo para comprender la dinámica cooperativa de los autores. Los datos obtenidos reportan bajo nivel de cooperación, de hecho, el número de contribuciones realizadas en solitario asciende a 27 artículos (49\%), además, el margen de cooperación con autores de otras instituciones y más aún con autores internacionales, es reducido. Al parecer, la escritura colaborativa todavía es una práctica que requiere mayor asiento entre el profesorado; en la red de autores, el nivel de densidad es de 0,04, lo que sugiere que $96 \%$ de las posibles vinculaciones entre autores no se concretan, adicionalmente, el número de intermediarios es pequeño, siendo únicamente siete los docentes que sirven de conexión entre otros autores. Así mismo, en la conformación de las redes no se aprecia una influencia de características como el sexo, la procedencia, formación o clasificación de los autores, tal y como se observa en los resultados del análisis de regresión.

La construcción de redes cooperativas sigue siendo una tarea por desarrollar en las distintas disciplinas de las ciencias sociales en Colombia, incluida la educación; los datos reportados en investigadores de la Universidad del Atlántico reiteran el panorama nacional, pues se han identificado redes de coautoría con una baja densidad y elevada distancia entre los investigadores en educación, incluso con necesidad de recorrer entre tres y siete distancias o caminos para poder establecer conexiones entre autores (ÁvilaToscano et al., 2019). En tal sentido, las universidades colombianas siguen llamadas a generar políticas que fomenten el espíritu colaborativo en la escritura científica, así como la construcción de lazos institucionales que fomenten la participación en el desarrollo de proyectos educativos. Del mismo modo, los investigadores deben considerar las ventajas que implica el trabajo colaborativo y la inserción en redes con otros articulistas, pues ha sido suficientemente demostrado que la colaboración científica, y en particular la de tipo internacional, aumenta la posibilidad de obtener recursos que financien la investigación, permite trabajar con investigadores de elevado prestigio y abordar los temas de análisis desde perspectivas transculturales (Puuska et al., 2014; Luna-Morales, 2012), y por supuesto, aumentar la calidad de los productos generados (Sancho et al., 2006).

Finalmente, el tercer elemento de estudio recae en la identificación de campos temáticos gracias a la aplicación del análisis de redes sociales para producir redes de términos clave en las contribuciones analizadas. En los resultados obtenidos emergen campos temáticos diversos, si bien los mismos no se muestran interconectados entre sí, a excepción de subconjuntos semánticos cuya vinculación es producto de términos clave de uso común en el campo educativo (estudiantes, autonomía), pero en general, las conexiones temáticas entre los subgrupos emergidos son pocas.

Estos resultados parecen indicar trabajo independiente entre autores pertenecientes a disciplinas específicas dentro del saber educativo, lo que se refrenda por la ya descrita baja conectividad en las redes de coautoría. En otras palabras, parece ser que la producción se genera desde los diferentes campos de aplicación educativa acorde con las disciplinas de estudio (matemáticas, lenguaje, etc.), y a partir de estas conexiones 
emergen subunidades temáticas claramente diferenciadas una de la otra. En el caso de la red de conocimiento abordada en la Universidad del Atlántico, se identifica un escenario analítico muy cercano al reportado en el contexto nacional, donde las principales temáticas descritas incluyen el papel de las TIC en la educación, las problemáticas sociales y su relación con la educación, e incluso el saber matemático se destaca como un campo particular (Ávila-Toscano, Romero-Pérez, Marenco-Escuderos et al., 2018).

El campo de estudios en educación generado en la Universidad del Atlántico incluye la educación matemática con grupos culturales y étnicos como el tópico más amplio, debido a la mayor productividad en la materia, así mismo, destacan los estudios enfocados en el uso de TIC y aspectos históricamente del interés en el ámbito educativo como el conocimiento pedagógico y las competencias del profesorado. Adicionalmente, se resalta un interés transversal por el estudio del contexto en los diferentes temas emergidos, de esta forma, el contexto sociocultural resulta relevante frente a disciplinas como la matemática, la literatura o el análisis de las problemáticas sociales del Caribe colombiano y sus implicaciones en el proceso educativo.

En resumen, los resultados del estudio muestran características generales de mucha importancia en la comprensión del sistema de producción y funcionamiento dentro un campo de conocimiento, tomando el caso particular de una unidad académica. Claramente, existirán diferencias entre las diversas instituciones en relación con el nivel de productividad y los lazos de cooperación, sin embargo, en términos generales los datos obtenidos constituyen un aporte importante para analizar el nivel de contribución que las universidades realizan a la investigación en educación. Adicionalmente, los resultados relacionados con los campos temáticos son similares a los recogidos en el contexto nacional, y sin duda llaman al fomento de la interdisciplinariedad que también puede fortalecerse a través de la configuración de redes entre autores e instituciones. Este tipo de trabajos son sustanciales para la revisión periódica de los aportes que desde la investigación se hace al saber educativo (Papadimitriou \& Kidman, 2012), además de permitir conocer ejes de estudios y estrategias de trabajo que aporten al posicionamiento de los diferentes actores en un área del conocimiento.

\section{Conclusión}

La investigación cienciométrica y bibliométrica constituye un aporte de mucha utilidad para estudiar el estado de desarrollo de la ciencia y sus diversas disciplinas, de ello también se han nutrido las ciencias de la educación gracias a la evidencia sobre líneas de investigación y tópicos de estudio que ofrecen un panorama general acerca de cómo los investigadores avanzan en ciertos campos temáticos o incluso, aquellos que pierden envergadura.

Este estudio ha procurado mostrar la importancia de conocer indicadores de producción 
dentro de una unidad académica de las ciencias de la educación, superando el simple reporte descriptivo de artículos para reconocer el papel de los autores en las dinámicas de relaciones cooperativas, así mismo, los métodos reticulares han aportado a la generación de campos de estudios dentro de los procesos investigativos que abordan la educación.

Resulta importante la generación de sinergias entre autores para lograr procesos de alto nivel que contribuyan a la consolidación de líneas y enfoques de estudios en el plano educativo, esto se verá enriquecido con la búsqueda de lazos que alienten la inserción de investigadores nacionales y extranjeros en los procesos de investigación surgidos desde lo regional, así mismo, la cooperación entre autores e instituciones y el trabajo en campos temáticos prolíficos ayuda a que los aportes al estado del arte en las ciencias de la educación sea significativo.

\section{Referencias}

Abramo, G., \& D'Angelo, C. (2014). How do you define and measure research productivity? Scientometrics, 101(2), 1129-1144. DOI: http://dx.doi.org/10.1007/s11192-014-1269-8

Aldana, E. (2010). El caso de Colombia. En: Santelices, B. (Coord.) El rol de las universidades en el desarrollo científico y tecnológico. Educación superior en Iberoamérica - Informe 2010. (pp. 163-167). España. RIL Editores.

Ávila-Toscano, J. H., y Marenco-Escuderos, A. (2016). Producción bibliométrica y redes de cooperación en la revista Psicología desde el Caribe. Psicología desde el Caribe, 33(1), 66-80. DOI: http://dx.doi.org/10.14482/psdc.33.1.8063

Ávila-Toscano, J. H., Marenco-Escuderos, A., \& Romero-Pérez, I. (2019). Redes de cooperación entre autores e instituciones en Ciencias Sociales dentro del modelo científico colombiano: comparación por género y área del conocimiento. Revista General de Información y Documentación, 29(1), 209-227. DOI: https://doi.org/10.5209/rgid.64545

Ávila-Toscano, J. H., Romero-Pérez, I., Saavedra, E., \& Marenco-Escuderos, A. (2018). Influencia de la producción de nuevo conocimiento y tesis de postgrado en la categorización de los grupos de investigación en Ciencias Sociales: árbol de decisiones aplicado al modelo científico colombiano. Revista Española de Documentación Científica, 41(4), 1-13. DOI: https://doi.org/10.3989/redc.2018.4.1547

Ávila-Toscano, J. H., Romero-Pérez, I., Marenco-Escuderos, A., \& Saavedra, E. (2018). Identification of research thematic approaches based on keywords network analysis in Colombian Social Sciences.Thomas, C. Data Mining. (pp. 145-164). 
Londres: IntechOpen. DOI: https://doi.org/10.5772/intechopen.76834

Borgatti, S., Everett, M., \& Freeman, L. (2002). Ucinet for Windows: Software for Social Network Analysis. Harvard, MA: Analytic Technologies.

Bracho-López, R., Maz-Machado, A., Gutiérrez-Arenas, P., Torralbo-Rodríguez, M., Jiménez-Fanjul, N., y Adamuz-Povedano, N. (2012). La investigación en Educación Matemática a través de las publicaciones científicas españolas. Revista Española de Documentación Científica, 35(2), 262-280. DOI: https://doi.org/10.3989/redc.2012.2.870

Brew, A., Boud, D., Namgung, S., Lucas, L., \& Crawford, K. (2016).Research productivity and academics' conceptions of research. Higher Education, 71(5), 681-697. DOI. https://doi.org/10.1007/s10734-015-9930-6

Bucheli, V., Díaz, A., Calderón, J., Lemoine, P., Valdivia, J., Villaveces, J., \& Zarama, R. (2012). Growth of scientific production in Colombian universities: an intellectual capital-based approach. Scientometrics, 91(2), 369-382. DOI: https://doi.org/10.1007/s11192-012-0627-7

CodePlex. (2013). NodeXL: Network Overview, Discovery and Exploration for Excel. Social Media Research Foundation. Recovered from: https://archive.codeplex.com/?p=nodexl

Colciencias - Departamento Administrativo de Ciencia, Tecnología e Innovación. 2017. Modelo de medición de grupos de investigación, desarrollo tecnológico o de innovación y reconocimiento de investigadores del sistema nacional de ciencia tecnología e innovación año 2017. Recuperado de: https://minciencias.gov.co/sistemas-informacion/modelo-medicion-grupos

Corchuelo, C. (2018). Visibilidad científica y académica en la web 2.0 Análisis de grupos de investigación de la Universidad de La Sabana. Información, Cultura y Sociedad, (38), 77-88. Recuperado de: https://bit.ly/2rtYPwT

Gantman, E. (2011). La productividad científica argentina en Ciencias Sociales: Economía, Psicología, Sociología y Ciencia Política en el CONICET (2004-2008). Revista Española de Documentación Científica, 34(3), 408-425. DOI: http://dx.doi.org/10.3989/redc.2011.3.829

González-Alcaide, G., \& Gómez Ferri, J. (2014). La colaboración científica: principales líneas de investigación y retos de futuro. Revista Española de Documentación Científica, 37(4), e062. DOI: http://dx.doi.org/10.3989/redc.2014.4.1186

González-Alcaide, G., Alonso-Arroyo, A., González de Dios, J., Sempere, A., Valderrama- 
Zurián, J., Aleixandre-Benavent, R. (2008). Redes de coautoría y colaboración institucional en Revista de Neurología. Revista de Neurología, 46(11), 642-651. DOI: https://doi.org/10.33588/rn.4611.2008158

Guzmán, M., \&Trujillo, J. (2013). Los mapas bibliométricos o mapas de la ciencia: una herramienta útil para desarrollar estudios métricos de información. Biblioteca Universitaria, 16(2), 95-108. Recuperado de: https://bit.ly/2TZtNKP

He, Q. (1999). Knowledge discovery through co-word analysis. Library Trends, 48(1), 133159. Recovered from: https://bit.ly/2Six9Gw

Hicks, D. (2004). The four literatures of social science. En: Handbook of quantitative science and technology research. (pp. 473-496). Kluber Academic. DOI: https://doi.org/10.1007/1-4020-2755-9_22

Hubert, L., \&Schultz, J. (1976). Quadratic Assignment as a general data analysis strategy. British Journal of Mathematical and Statistical Psychology, 29(2), 190-241. DOI: https://doi.org/10.1111/j.2044-8317.1976.tb00714.x

Leydesdorff, L., \& Rafols, I. (2009). A global map of science based on the ISI subject categories. Journal of the American Society for Information Science and Technology, 60(2), 348-362. DOI: https://doi.org/10.1002/asi.20967

Luna-Morales, M. (2012). La colaboración científica y la internacionalización de la ciencia mexicana de 1980 a 2004. Investigación Bibliotecológica, 26(57), 103-129. Recuperado de: https://bit.ly/2TVELRm

Maz-Machado, A., Bracho-López, R., Torralbo-Rodríguez, M., Gutiérrez-Arenas, M., \& Hidalgo-Ariza, M. (2011). La investigación en Educación Matemática en España: los simposios de la SEIEM. PNA, 5(4), 163-184. Recuperado de: https://bit.ly/2zehs]b

Maz-Machado, A., Jiménez-Fanjul, N., \& Villarraga, E. (2016). La producción científica colombiana en SciELO: un análisis bibliométrico. Revista Interamericana de Bibliotecología, 39(2), 111-119. DOI: https://doi.org/10.17533/udea.rib.v39n2a03

Miguel, S., Chinchilla-Rodríguez, Z., González, C., \& Moya Anegón, F. (2012). Analysis and visualization of the dynamics of research groups in terms of projects and co-author edpublications. A case study of library and information science in Argentina. Information Research, 17(3), 5-24. Recovered from: https://bit.ly/2YNNw40

Papadimitriou, F., \& Kidman, G. (2012). Statistical and scientometric analysis of international research in geographical and environmental education. Journal of International Research in Geographical and Environmental Education, 21(1), 11-20. DOI: https://doi.org/10.1080/10382046.2012.639153 
Porter, A., \& Rafols, I. (2009). Is science becoming more interdisciplinary? Measuring and mapping six research fields over time. Scientometrics, 81(1), 719-745. DOI: https://doi.org/10.1007/s11192-008-2197-2

Puuska, H., Muhonen, R., \&Leino, Y.(2014). International and domestic co-publishing and their citation impact in different disciplines. Scientometrics, 98(2), 823-839. DOI: https://doi.org/10.1007/s11192-013-1181-7

Rosario-Sierra, M., Sánchez-Perdomo, R., Herrera-Vallejera, D., \& Rodríguez-Sánchez, Y. (2017). Patrón de citas de la producción científica en Ciencias Sociales y Humanidades. Un análisis a partir de Scopus (2000- 2012). Ciencias de la Información, 48(1), 37-44. Recuperado de: https://bit.ly/2HjLuzN

Russell, J., Ainsworth, S., \& Narváez-Berthelemot, N. (2006). Colaboración científica de la Universidad Nacional Autónoma de México (UNAM) y su política institucional. Revista Española de Documentación Científica, 2(1), 56-73. DOI: https://doi.org/10.3989/redc.2006.v29.i1.287

Spinak, E. (2001). Indicadores cienciométricos. ACIME, 9(4), 16-18. Recuperado de: https://bit.ly/2Np6mJv

Sancho, R., Morillo, F., De Filippo, D., Gómez, I., \& Fernández, M. (2006). Indicadores de colaboración científica inter-centros en los países de América Latina. Interciencia, 31(4), 284-292.

Shin, J., \& Cummings, W.(2010). Multilevel analysis of academic publishing across disciplines: research preference, collaboration, and time on research. Scientometrics, 85(2), 581-594. DOI: https://doi.org/10.1007/s11192-010-0236-2

Silva, F., Viana, M., Travencolo, B., \&Costa, L. (2011). Investigating relationships within and between category networks in wikipedia. Journal of Informetrics, 5(3), 431438. DOI: https://doi.org/10.1016/j.joi.2011.03.003

Vélez-Cuartas, G., Lucio-Arias, D., \& Leydesdorff, L. (2016). Regional and global science: Publications from Latin America and the Caribbean in the SciELO Citation Index and the Web of Science. El Profesional de la Información, 25(1), 35-46. DOI: https://doi.org/10.3145/epi.2016.ene.05 